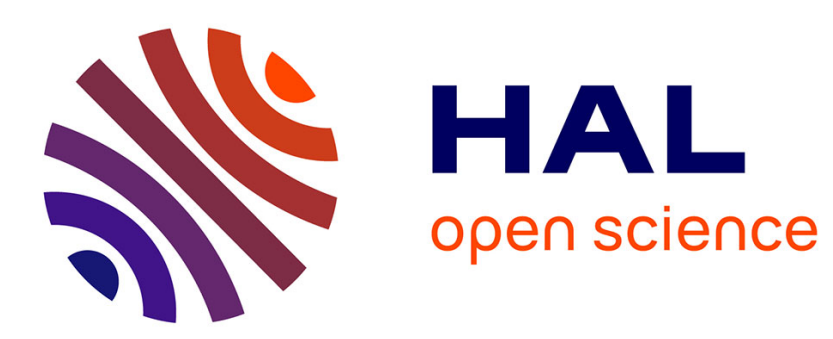

\title{
Impulse Response Measurements using MLS Technique on Nonsynchronous Devices
}

Antonin Novak, František Rund, Petr Honzík

\section{To cite this version:}

Antonin Novak, František Rund, Petr Honzík. Impulse Response Measurements using MLS Technique on Nonsynchronous Devices. Journal of the Audio Engineering Society, 2016, 64 (12), pp.978-987. 10.17743/jaes.2016.0050 . hal-02504349

\section{HAL Id: hal-02504349 \\ https://hal.science/hal-02504349}

Submitted on 10 Mar 2020

HAL is a multi-disciplinary open access archive for the deposit and dissemination of scientific research documents, whether they are published or not. The documents may come from teaching and research institutions in France or abroad, or from public or private research centers.
L'archive ouverte pluridisciplinaire HAL, est destinée au dépôt et à la diffusion de documents scientifiques de niveau recherche, publiés ou non, émanant des établissements d'enseignement et de recherche français ou étrangers, des laboratoires publics ou privés. 


\title{
Impulse Response Measurements using MLS Technique on Nonsynchronous Devices
}

\author{
Antonin Novak ${ }^{1 *}$, Frantisek Rund ${ }^{2}$, Petr Honzik ${ }^{3}$ \\ ${ }^{1}$ Laboratoire d'Acoustique de I'Université du Mans, LAUM - UMR 6613 CNRS, Le Mans Université, Avenue Olivier \\ Messiaen, 72085 LE MANS CEDEX 9, France \\ ${ }^{2}$ Czech Technical University in Prague, Faculty of Electrical Engineering, Prague, Czech Republic \\ ${ }^{3}$ Czech Technical University in Prague, Faculty of Transportation Sciences, Prague, Czech Republic \\ *antonin.novak@univ-lemans.fr \\ *https: //ant-novak. com
}

\begin{abstract}
Maximum-length sequences (MLS) are widely used for measurement of impulse responses of linear time-invariant systems in acoustic and audio systems. It is usually believed that one of the drawbacks of the MLS technique is a requirement on a synchronization of the devices used for the generation and acquisition of the MLS signals. The study presented in this paper shows that the MLS technique can easily be improved and applied to devices that are not synchronous, or even operating at different sampling frequencies. To show the efficiency of the proposed modification to MLS technique we provide several experiments such as a measurement with devices working at $44.1 \mathrm{kHz}$ on the generation side and $96 \mathrm{kHz}$ on the acquisition side, or a measurement of the frequency response function of a cheap mobile phone in which the synchronous clocking would be impossible to realize.
\end{abstract}

The archived file is not the final published version of the article A. Novak, F. Rund \& P. Honzik (2016), "Impulse Response Measurements using MLS Technique on Nonsynchronous Devices", Journal of the Audio Engineering Society. Vol. 64(12), pp. 978-987.

The definitive publisher-authenticated version is available online at https://doi.org/10.17743/jaes.2016.0050, Readers must contact the publisher for reprint or permission to use the material in any form. 


\section{INTRODUCTION}

One of the most important and most common tasks in audio and acoustics is the measurement of the impulse response (IR) or of its related frequency response function (FRF). Considering the system under test is a linear time-invariant (LTI) system, the IR is defined as the output of the system when a Dirac impulse is applied to the input. Besides the IR measurement method, that is exciting the system under test with an impulse or with a sequence of pulses, there exists many different and more robust techniques to obtain the impulse response. In general, these techniques are based on wide-band excitation signals, such as chirps, swept-sines, and random or pseudo-random signals.

The maximum-length sequence (MLS) technique, a member of pseudo-random signals family, has become popular in audio and acoustic measurements few decades ago thanks to the work of Schroeder [1] and later published more detailed studies such as $[2,3,4,5]$. One of its main and important advantages is a high signal-to-noise ratio (SNR) and its robustness against impulsive or non-stationary disturbances [6]. On the other hand, contrary to swept-sine signals $[7,8]$, the MLS technique can not properly deal with nonlinear or time-variant systems and exhibits negative effects such as distortion artifacts and increased noise [5, 9, 10, 11, 12, 13, 14, 15].

Moreover, as discussed in [7], the MLS technique requires that the device used for the signal generation is synchronized with the device employed for acquiring the response of the system. The excellent performance of the MLS technique is dramatically degraded if the sampling frequencies of the D/A and the A/D converters are not exactly the same. The consequent artifacts due to non-synchronization, already described in [5], have been studied in $[11,16]$ and lead to an important decrease of SNR or to an inability to extract the impulse response when the sampling frequencies of both devices differ.

However, for some practical cases, it might be very inconvenient to have the generation and acquisition in the synchronous clocking mode (e.g. large room impulse response measurements where the source and the microphone are located far from each other, measurement of broadcasting or VoIP chains, or simply using audio interfaces that do not support the clock synchronization). We show in this paper, that the MLS technique can be easily modified to work correctly even if the device used for the signal generation is not synchronized with the device employed for the signal acquisition (so-called asynchronous clocking mode).

We recall the basics of the MLS technique in section 2. The principles of the synchronous and asynchronous clocking modes are explained in section 3 and the difficulties of MLS technique when used under the asynchronous clocking mode are detailed in section 4 including an example of MLS measurements in both modes. To overcome these difficulties a solution is proposed in section 5 and validated by several experiments presented in section 6 .

\section{MLS TECHNIQUE: A BRIEF REVIEW}

The maximum-length sequence is a pseudo-random sequence of binary numbers 0 and 1 usually transformed to a bipolar signal of amplitude $\pm V_{0}[5]$. The most important property of this sequence is that its circular auto-correlation function results in a train of Kronecker delta functions $\delta(n)$ shifted by a DC-component as

$$
R_{x x}^{\circ}[n]=\sum_{m=-\infty}^{\infty} \delta[n+m L]-\frac{1}{L+1}
$$

the period $L=2^{N}-1$ being given by the order $N$ of the sequence [17]. Consequently, a circular cross-correlation between the original sequence $x[n]$ and the sequence $y[n]$ passed through the system under test results in the train of IRs of the system,

$$
R_{x y}^{\circ}[n]=\sum_{m=-\infty}^{\infty} h[n+m L]-\frac{1}{L+1} \sum_{i=0}^{L-1} h[i] .
$$


Therefore, the IR of the system under test can be obtained using a circular cross-correlation of the MLS $x[n]$ and the recorder output $y[n]$. The practical implementation of the circular correlation of very long sequences $(\mathrm{N}=20)$ is nowadays a very fast task using either a Walsh-Hadamard transform or applying the correlation directly in the frequency domain using the Fast Fourier Transform as

$$
R_{x \bar{y}}^{\circ}[n]=\frac{1}{L} \sum_{k=0}^{L-1} X[k] Y^{*}[k] e^{j \frac{2 \pi k n}{L}},
$$

where $X[k]$ and $Y[k]$ are the Discrete Fourier Transforms (DFT) of $x[n]$ and $y[n]$, and where * denotes the complex conjugate.

It is a good practice to use at least two MLS periods to perform the measurement. One full MLS period is used to stabilize the system so it is steady state, the second one being used to actually make the measurement [3]. It is also common to apply several MLS periods to the linear system under test and then average the measured impulse response. The order of the MLS can also affect the performance of this technique. It has been demonstrated in [18], that the signal-to-noise ratio of the measured impulse response increases by $3 \mathrm{~dB}$ when the period length of the MLS sequence is doubled.

More details about the generation of the MLS and the theoretical background can be found in [5].

\section{SYNCHRONOUS VS. ASYNCHRONOUS CLOCKING MODE}

When it comes to measuring the IR of a linear system, in most cases a dedicated digital device that generates the output signal and records the input signal at the same time in a synchronous clocking mode $[19,20,21]$ is used. In some cases, however, it is difficult or even impossible to ensure a perfect synchronization between the generation and the acquisition of these signals. Such a mode is called an asynchronous clocking mode and is usually not recommended to be used for MLS technique [7, 10, 22].

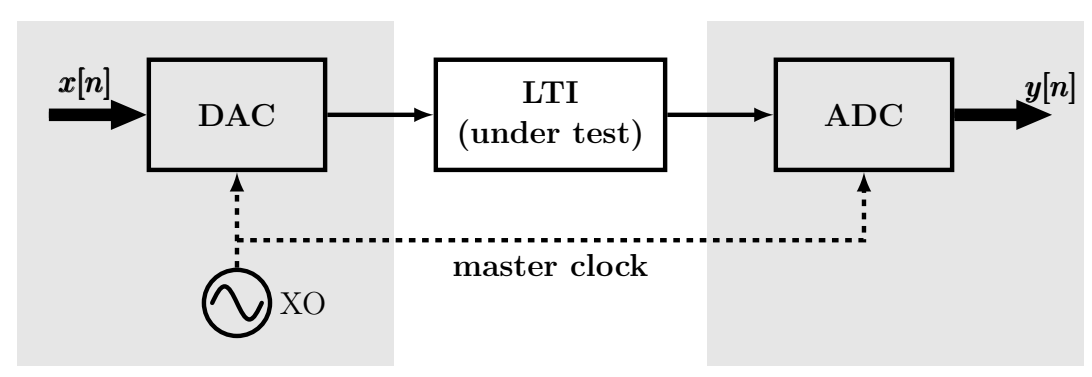

(a) In the synchronous clocking mode, the DAC on the generating side and the ADC on the acquiring side operate at the same data rates.

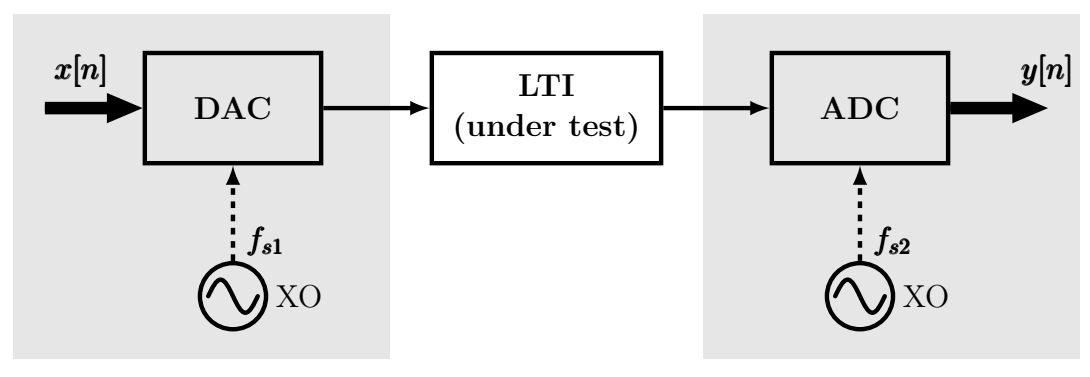

(b) In the asynchronous clocking mode, the DAC on the generating side and the ADC on the acquiring side operate at two different data rates.

Figure 1. Synchronous vs. asynchronous clocking mode. 
Indeed, a typical digital audio system used for signal generation and/or signal acquisition employs an Analog-toDigital and/or Digital-to-Analog converter (ADCs and DACs) equipped with a crystal oscillator (XO) to generate a clock signal with the desired sampling frequency $f_{s}$. Since no oscillator can perfectly generate the exact sampling frequency, there is always a small clock error that may cause a few difficulties in digital signal processing. The clock error can be divided into three main categories. The first one, an accuracy error, describes how correctly the generated sampling frequency $\tilde{f}_{s}$ fits $f_{s}$. The second one, a stability error, is represented by the time-variation of the $\tilde{f}_{s}(t)$ and finally the jitter error refers to variations within one period of the clock signal.

While in the synchronous clocking mode there is only one master clock driven by a single oscillator (Fig. 1a), when working in an asynchronous clocking mode, the sampling frequencies of the generation and the acquisition devices are driven by two different oscillators whose frequencies are close but not identical (Fig. 1b). The difference between these two frequencies due to different clock errors of both oscillators might be very small, of the order of tenths or even hundredths of a percent, but even such a small inaccuracy of sampling clock may result in a huge error of impulse response estimation using the MLS signals [7, 10, 22, 16].

\section{MLS PROBLEMS WITH AN ASYNCHRONOUS MODE}

As demonstrated in [5, 10], the problem of time-variation and jitter in MLS signals results in an decreased signalto-noise ratio. When using the MLS technique with an asynchronous clocking mode, the accuracy error of both clock signals (Fig. 1b) leads to two slightly different sampling frequencies $f_{s 1}$ and $f_{s 2}$ and consequently to a huge error of IR estimation.

\subsection{Experiment with the asynchronous clocking mode}

To demonstrate the problems with the asynchronous clocking mode, we use two high-quality audio interfaces of the same series (RME Fireface 400), one for signal generation and the other one for signal acquisition. Two experiments are then considered: the first one in the synchronous and the second one in the asynchronous clocking mode. In both experiments, we connect the audio output of the first device to the audio input of the second one, so that the measured FRF should be equal to 1 in a very large frequency band and the IR should be very close to the delta function. These two experiments are depicted in Fig. 1 where the considered LTI system is a short wire.

In the first experiment the internal clock of the generating device is set to $f_{s}=96 \mathrm{kHz}$ and its clock signal is connected to the clock input of the second device (Fig. 1a) corresponding to the synchronous clocking mode. The

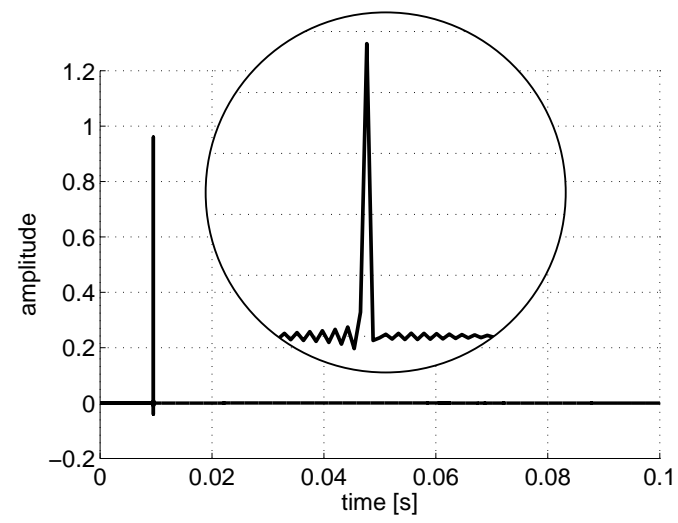

(a)

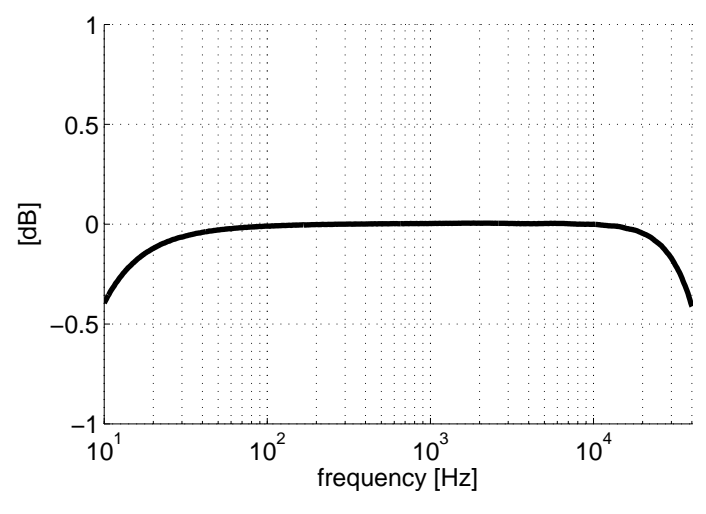

(b)

Figure 2. Estimated IR (a) and FRF (b) using the MLS technique (order 20). The master clock sampling frequency is set to $96 \mathrm{kHz}$ and both devices are working in the synchronous clocking mode. 


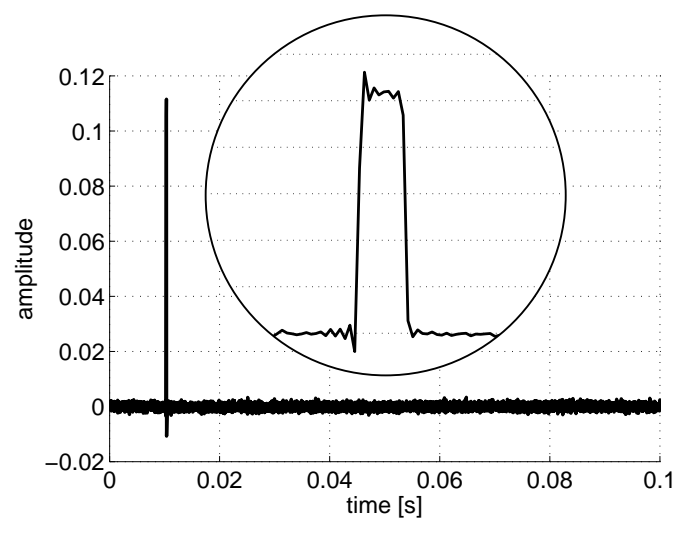

(a)

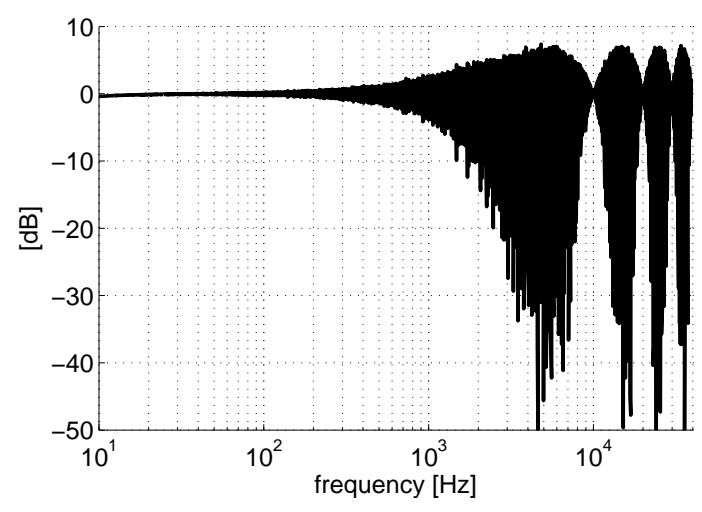

(b)

Figure 3. Estimated IR (a) and FRF (b) using the MLS technique (order 20). Clock frequencies of both devices are independent (asynchronous clocking mode) and are set to $\simeq 96 \mathrm{kHz}$.

MLS signal (order $\mathrm{N}=20$ ) is used to measure the system under test and the IR is calculated using the FFT. The result presented in Fig. 2a shows the IR of the measured system which is very close to the delta function and the FRF presented in Fig. $2 \mathrm{~b}$ shows a very flat response within a large frequency band. The small drop at very low and very high frequencies, not exceeding $0.5 \mathrm{~dB}$, is due to the DC-protecting and anti-aliasing filter respectively.

The second experiment is similar to the first one, except that the clock signal is disconnected and each of the RME Fireface 400 interfaces is set to its internal clock at $f_{s} \simeq 96 \mathrm{kHz}$. Such a configuration is depicted in Fig. 1b and corresponds to the asynchronous clocking mode. The estimated IR is depicted in Fig. 3a, exhibiting an increase of the noise level, as well as a decrease of the peak value (pay attention to the y-axis of Fig. 2a and Fig. 3a). The zoom near the maximum energy of the IR, depicted in Fig. 3a, shows an important degradation of the estimated IR. The FRF depicted in Fig. 3b similarly highlights a huge degradation equivalent to an added noise especially at higher frequencies (once again pay attention to the y-axis of Fig. 2b and Fig. 3b).

Even though two very high-quality devices with high precision clock sources have been used for these experiments, the IR and consequently the FRF estimated in the asynchronous clocking mode are so degraded that the MLS technique seems to be inapplicable.

\subsection{Analysis of the asynchronous clocking mode}

In order to understand the reason why the estimated IR in asynchronous clocking mode differs from the expected one, we analyze the problem in both time and frequency domain (Fig. 4).

First, an MLS $x[n]$ with length $L=31$ (order $N=5$ ) and its DFT are depicted in Fig. 4a. The modulus of the DFT of an MLS is $\sqrt{1 / L}$ at DC (and at multiples of $L$ ) and $\sqrt{(L+1) / L}$ for the other spectral lines (Fig. 4a) and has the pseudo-random phase uniformly distributed over its range of 0 to $2 \pi$ at all other frequencies.

The digital-to-analog conversion of the MLS sequence is, for the sake of simplicity, realized using an ideal reconstruction (e.g. ideal band-limited interpolation) working at sampling frequency $f_{s 1}$ (Fig. $4 \mathrm{~b}$ ).

The reconstructed analog signal $x(t)$ is then converted back to $y[n]$ using an ideal analog-to-digital converter working at sampling frequency $f_{s 2}$, in our example (Fig. 4c) $f_{s 2}>f_{s 1}$. Since the time period of the original MLS sequence is

$$
T=\frac{L}{f_{s 1}}
$$

and since the time length of the MLS period is the same for both $x[n]$ and $y[n]$ sequences, the number of samples 
of $y[n]$ within one period $T$ is now greater than $L$ by $\Delta L$ samples, satisfying

$$
T=\frac{L+\Delta L}{f_{s 2}},
$$

or in other words

$$
\Delta L=L\left(\frac{f_{s 2}}{f_{s 1}}-1\right)
$$

Finally, the sequence $y[n]$ is truncated to $L$ samples in order to match the number of samples of the original MLS $x[n]$ and the Walsh-Hadamard transform or the Fast Fourier Transform is then applied to estimate the IR. However, truncation of the sequence $y[n]$ to $L$ samples is equivalent to a multiplication by a rectangular function of length $L$ leading to a convolution with a sinc function in frequency domain. The DFT of the truncated sequence $y[n]$ depicted in Fig. 4d looks like a random distribution of amplitudes across all frequencies. A statistical analysis of the truncated sequence $y[n]$ in frequency domain is provided in Appendix. It shows that the degradation is more important at higher frequencies as shown in the results of the asynchronous measurements (Fig. 3).

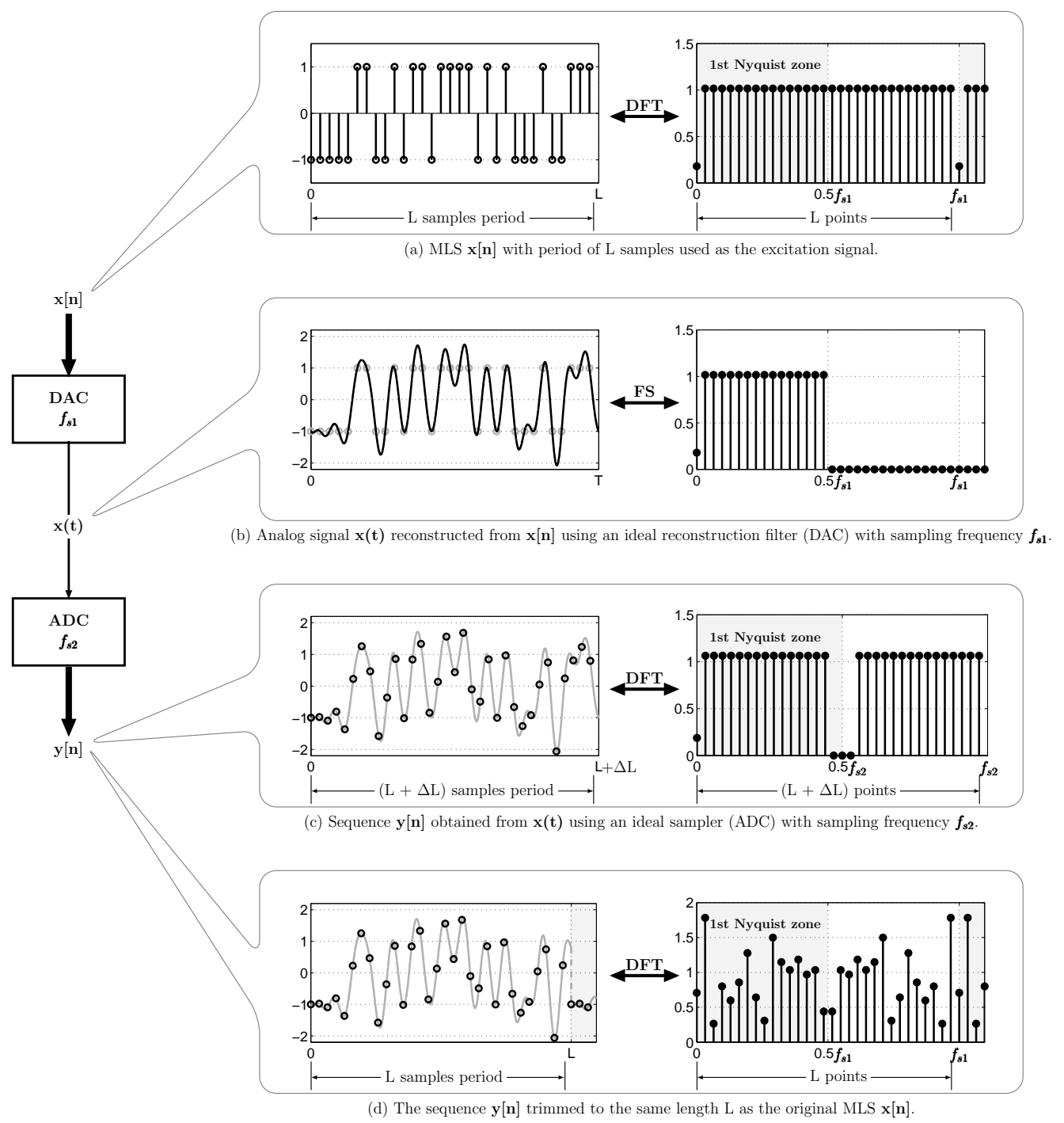

Figure 4. Graphical representation of the analysis of the MLS asynchronous problem in both the time and the frequency domain. 


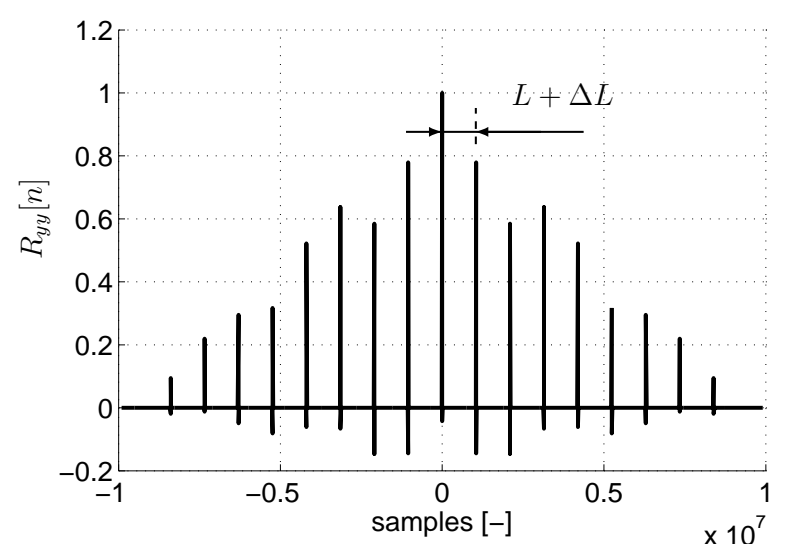

Figure 5. The autocorrelation function $R_{y y}[n]$ of the acquired response to the MLS signal measured in the asynchronous clocking mode. The distance between peaks, equivalent to the period of the acquired signal, is by $\Delta L$ samples longer than the period $L$ of the MLS.

\section{PROPOSED SOLUTION}

It has been shown in section 4, that if we measure the system under test in the so-called asynchronous clocking mode, the number of samples of each period may differ from the number of samples of one period of the original MLS. Consequently, the estimated IR of the system under test is degraded. We show in this section that it is possible to obtain the IR correctly with almost no degradation or added noise by implementing a simple modification to the MLS method.

The first step of the proposed solution consists in estimating the number of samples of each period of the recorded response to the MLS. Suppose, for example, that the excitation signal $x[n]$ consists of at least three periods of the MLS. Cutting off the beginning of the output signal $y[n]$ (used to stabilize the system [3]), there remains at least two periods of the MLS output from which the length of the period can be estimated using the auto-correlation function. An example of an auto-correlation function of the MLS response of an asynchronous measurement $(\mathrm{N}=$ 20, 9 periods of MLS) is depicted in Fig. 5 . The length difference $\Delta L$ can be then easily estimated form the position of the peaks of the auto-correlation function.

The estimated length in the case of the particular example presented in section 4.1 is longer by $\Delta L=10$ samples than the length of the original MLS $\left(L=2^{20}-1\right)$. We can then conclude that the sampling frequency of the acquisition device is higher than the one of the excitation device and can be calculated as

$$
f_{s 2}=f_{s 1} \frac{L+\Delta L}{L}
$$

Note also that the offset between both sample frequencies $f_{s 2}$ and $f_{s 1}$ is, for this particular example, less than $1 \mathrm{~Hz}$ for $96 \mathrm{kHz}$ sampling rate (less than $0.001 \%$ ). Unfortunately, even such a small difference is high enough to corrupt the estimated IR presented in Fig. 3.

The second step of the proposed method is to take into account the length difference $\Delta L$ of both periodic sequences $x[n]$ and $y[n]$, and to perform the cyclic correlation of them. This can be done either in the time domain, re-sampling or interpolating the output signal $y[n]$ of length $L+\Delta L$ to a sequence of length $L$, or in the frequency domain by adding or removing samples from the spectra. Being now aware of the length difference $\Delta L$ estimated in the first step, we can remove $\Delta L$ points of the DFT of $y[n]$ and compute the circular correlation over $L$ points 
directly in the frequency domain. The DFTs of the sequences $x[n]$ and $y[n]$ being defined as

$$
\begin{gathered}
X[k]=\sum_{n=0}^{L-1} x[n] e^{-j \frac{2 \pi k n}{L}}, \\
Y[k]=\sum_{n=0}^{L+\Delta L-1} y[n] e^{-j \frac{2 \pi k n}{L+\Delta L}},
\end{gathered}
$$

the removal of $\Delta L$ points of the DFT can be done as

$$
\bar{Y}[k]=\left\{\begin{array}{ll}
Y[k], & 0 \leq k \leq \frac{L-1}{2} \\
Y^{*}[L-k], & \frac{L+1}{2} \leq k \leq L-1
\end{array},\right.
$$

considering $\Delta L>0$. We need to keep in mind that the sample spacing of both DFTs is equivalent to $1 / T$ and thus the $L$-point periodic DFT refers to sampling frequency $f_{s 1}$ to satisfy Eq. (4). Alternatively, $\Delta L$ zeros can be added to the spectra of $x[n]$ and then use the $(L+\Delta L)$-point periodic DFT to estimate the IR at sampling frequency $f_{s 2}$ (Eq. (5)). If $\Delta L<0, \Delta L$ points need to be removed from the DFT of $x[n]$, or alternatively $\Delta L$ zeros need to be added to the DFT of $y[n]$.

All these operations can be easily done in the frequency domain with almost no added operational cost. To obtain the desired impulse response, the circular cross-correlation is performed in the frequency domain using Eq. (3).

\section{EXPERIMENTS}

In order to validate the theory described in this paper, we present the results of several experiments of IR estimation using the MLS technique in the asynchronous clocking mode.

The first experiment is the one presented in section 4.1. The estimated IR using the MLS technique presented in Fig. 3 shows an important degradation due to the non-synchronization of the generating and acquisition devices. Using the modification of the MLS technique described in section 5 , we estimate the sample difference $\Delta L=10$ and remove them from the output spectra $Y[k]$ to get $\bar{Y}[k]$ as described in Eq. (10). Both the IR and FRF estimated using the proposed technique are depicted in Fig. 6. Note that compared to the synchronous clocking mode measurements (Fig. 2), the results presented in Fig. 6 in the asynchronous clocking mode corrected by the proposed method are almost the same ones. A tiny increase of noise is noticeable at higher frequencies (variation of $\pm 0.03 \mathrm{~dB}$ ) probably due to the jitter effect.

In the next experiment, we measure once again the same system under test, i.e. two audio interfaces RME Fireface 400 with the analog output of the first one connected directly to the analog input of the second one, the sampling frequency being this time $f_{s 1}=44.1 \mathrm{kHz}$ and $f_{s 2}=96 \mathrm{kHz}$. The results presented in Fig. 7 show that the proposed method can cope with two completely different sampling frequencies. The increased noise at high frequencies visible in Fig. $7 \mathrm{~b}$ is slightly higher than in the previous experiment, but does not exceed $\pm 0.1 \mathrm{~dB}$.

The last experiment is one of those in which the synchronous clocking mode can not be provided and highlights the robustness of the proposed method. A low-cost mobile phone with an Android system is loaded with an MLS (order 18) in the wav file $\left(f_{s 1}=48 \mathrm{kHz}\right)$ and the MLS is played a) through the phones output and a $3.5 \mathrm{~mm}$ to $6.35 \mathrm{~mm}$ mono jack cable to recording device (Fig. 8a) and b) through the loudspeaker of the mobile phone and recorded using a 1/8-inch pressure microphone (Grass 40DP) (Fig. 9a). The sampling frequency of the recording device (RME Fireface 400) is set to $48 \mathrm{kHz}$ and the proposed method is applied to cope with the asynchronous clocking mode. 


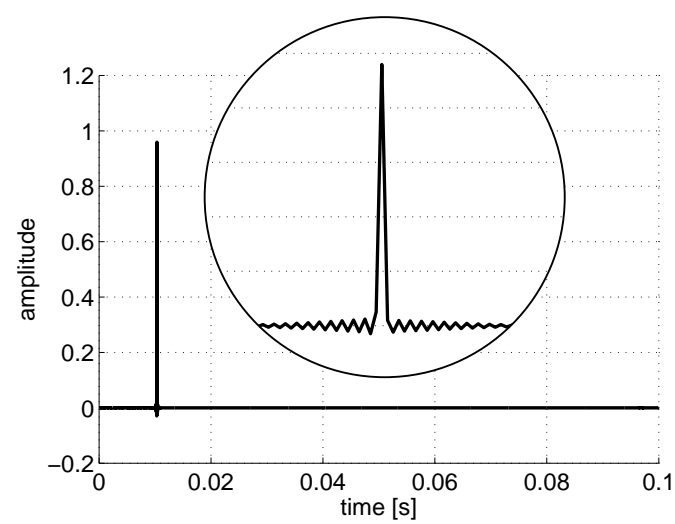

(a)

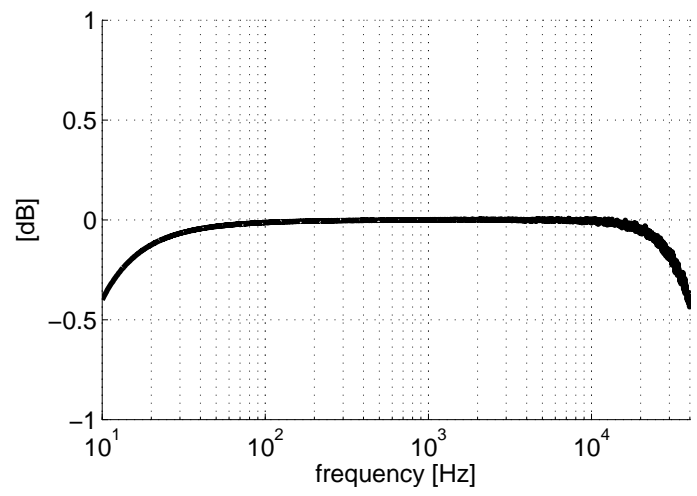

(b)

Figure 6. Estimated IR (a) and FRF (b) using the modified MLS technique (order 20). Clock frequencies of both devices are independent (asynchronous clocking mode) and are set to $\simeq 96 \mathrm{kHz}$ as in Fig. 3 .

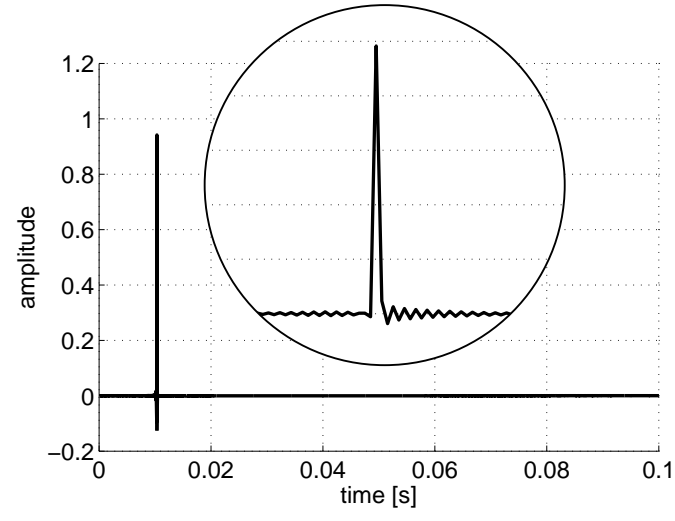

(a)

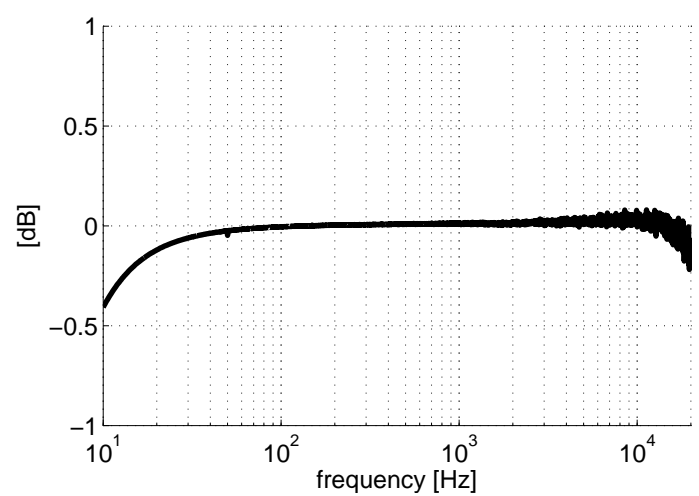

(b)

Figure 7. Estimated IR (a) and FRF (b) using the modified MLS technique (order 18). Clock frequencies of both devices are independent (asynchronous clocking mode) and are set to $44.1 \mathrm{kHz}$ on the generation side and to $96 \mathrm{kHz}$ at the acquisition side.

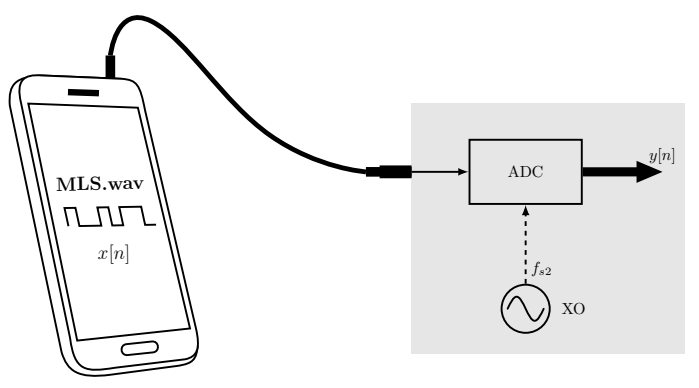

(a)

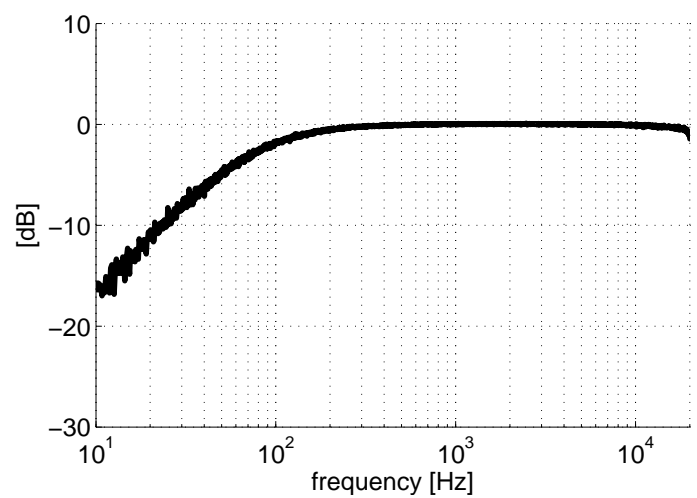

(b)

Figure 8. Block schema and the estimated FRF of the experiment with a low-cost mobile phone connected through the audio output to an acquisition device, both working at sampling frequency $48 \mathrm{kHz}$ : a) block schema of the MLS measurement, b) the corresponding FRF. 

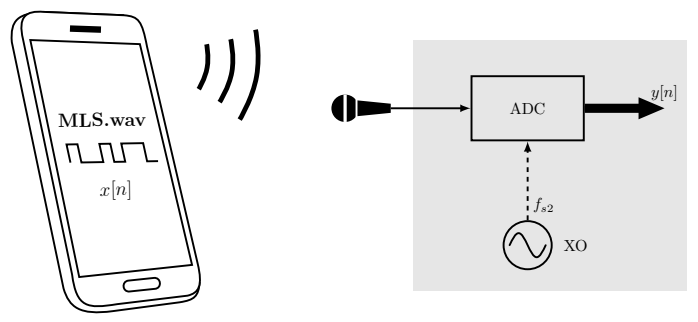

(a)

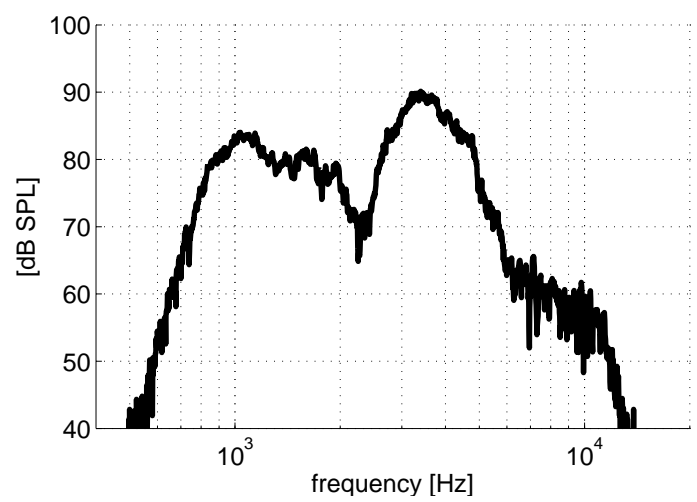

(b)

Figure 9. Block schema and the estimated FRF of the measurement of the acoustic characteristics of a low-cost mobile phone: a) block schema of the MLS measurement, b) the corresponding FRF.

The estimated FRF of the mobile phone audio output is presented in Fig. 8b. Although it is not possible to perform the measurement in synchronous clocking mode to provide a quantitative evaluation, it is clear from Fig. $8 \mathrm{~b}$ that the estimated FRF is meaningful and represents a high-pass filter with the cut-off frequency near $100 \mathrm{~Hz}$. The FRF is flat between $200 \mathrm{~Hz}$ and $20 \mathrm{kHz}$ with very small amount of noise $( \pm 0.1 \mathrm{~dB})$. The acoustical output of the mobile phone characterized by the estimated FRF (Fig. 9b) shows a typical response of a miniature loudspeaker used for mobile phones [23]. Since there is no way to synchronize the mobile phone with the acquisition device (except a very complicated hardware hacking of the phone), it is not possible to obtain such a results using the MLS technique without the modification proposed in this paper.

\section{DISCUSSION AND CONCLUSION}

The maximum-length sequence (MLS) technique is one of the most used methods for impulse response measurements in audio engineering. It is believed that the MLS technique requires a synchronization of the devices used for the generation and the acquisition (so-called synchronous clocking mode). When applied in so-called asynchronous clocking mode, i.e. the devices used for the generation and the acquisition are operating at two independent sampling frequencies, the estimated impulse response using the MLS technique is corrupted. We have shown that even when using high quality audio interfaces whose sampling frequency difference has been estimated to be less than $1 \mathrm{~Hz}$ for $96 \mathrm{kHz}$ sampling rate, the estimated impulse response is drastically degraded. Since the frequency offset of cheaper sound devices may be in the range of up to $10 \mathrm{~Hz}$ their usage in asynchronous mode may lead to an inability to extract the impulse response from the MLS measurements.

We show in this paper, that applying few modifications to the existing MLS technique that are easy to be implemented and which do not require any excessive computational cost, the MLS technique can also be used in the asynchronous clocking mode. As such, it can be extremely useful in situations where the generating and the acquisition devices can not be synchronized.

Several experiments given in this paper show the robustness of the modified MLS technique. Not only can the proposed method deal with the data obtained from the measurements on two audio asynchronous interfaces both set up to the same sampling frequency, it can also deal with two completely different sampling frequencies such as $44.1 \mathrm{kHz}$ and $96 \mathrm{kHz}$ as shown in one of the experiments in this paper. 


\section{Acknowledgement}

This research was funded by the Region Pays de la Loire within the Le Mans Acoustic Project and partially supported by the Grant Agency of the Czech Technical University in Prague, grant No. SGS15/226/OHK2/3T/16. The authors are grateful to prof. Laurent Simon from LAUM for helpful discussions.

\section{References}

[1] M. R. Schroeder, "Integrated-impulse method measuring sound decay without using impulses," The Journal of the Acoustical Society of America, vol. 66, no. 2, pp. 497-500, 1979.

[2] J. Borish and J. B. Angell, "An efficient algorithm for measuring the impulse response using pseudorandom noise," Journal of the Audio Engineering Society, vol. 31, no. 7/8, pp. 478-488, 1983.

[3] D. D. Rife and J. Vanderkooy, "Transfer-function measurement with maximum-length sequences," Journal of the Audio Engineering Society, vol. 37, no. 6, pp. 419-444, 1989.

[4] C. Dunn and M. J. Hawksford, "Distortion immunity of MLS-derived impulse response measurements," Journal of the Audio Engineering Society, vol. 41, no. 5, pp. 314-335, 1993.

[5] J. Vanderkooy, "Aspects of MLS measuring systems," Journal of the Audio Engineering Society, vol. 42, no. 4, pp. 219-231, 1994.

[6] J. L. Nielsen, "Improvement of signal-to-noise ratio in long-term MLS measurements with high-level nonstationary disturbances," Journal of the Audio Engineering Society, vol. 45, no. 12, pp. 1063-1066, 1997.

[7] A. Farina, "Simultaneous measurement of impulse response and distortion with a swept-sine technique," in Audio Engineering Society Convention 108, Audio Engineering Society, 2000.

[8] A. Novak, P. Lotton, and L. Simon, "Synchronized Swept-Sine: Theory, Application, and Implementation," Journal of the Audio Engineering Society, vol. 63, no. 10, pp. 786-798, 2015.

[9] M. Vorländer and M. Kob, "Practical aspects of MLS measurements in building acoustics," Applied Acoustics, vol. 52, no. 3, pp. 239-258, 1997.

[10] P. Svensson and J. L. Nielsen, "Errors in MLS measurements caused by time variance in acoustic systems," Journal of the Audio Engineering Society, vol. 47, no. 11, pp. 907-927, 1999.

[11] D. G. Ciric and M. A. Milošević, "Influence of MLS signal recording on various mediums," in Telecommunications in Modern Satellite, Cable and Broadcasting Service, 2003. TELSIKS 2003. 6th International Conference on, vol. 2, pp. 685-688, IEEE, 2003.

[12] J. P. Paulo, C. R. Martins, and J. B. Coelho, "A hybrid MLS technique for room impulse response estimation," Applied Acoustics, vol. 70, no. 4, pp. 556-562, 2009.

[13] S. Herzog and A. Potchinkov, "Audio testing by means of families of binary correlation sequences," Journal of the Audio Engineering Society, vol. 60, no. 11, pp. 913-925, 2012.

[14] M. Garai and P. Guidorzi, "Sound reflection measurements on noise barriers in critical conditions," Building and Environment, vol. 94, pp. 752-763, 2015. 
[15] P. Guidorzi, L. Barbaresi, D. DÓrazio, and M. Garai, "Impulse Responses Measured with MLS or Swept-Sine Signals Applied to Architectural Acoustics: An In-depth Analysis of the Two Methods and Some Case Studies of Measurements Inside Theaters," Energy Procedia, vol. 78, pp. 1611-1616, 2015.

[16] D. G. Ciric, "Synchronisation and excitation for MLS technique implementation in room acoustics," in Telecommunications in Modern Satellite, Cable and Broadcasting Services, 1999. 4th International Conference on, vol. 2, pp. 653-656, IEEE, 1999.

[17] W. Davies, "Generation and properties of maximum length sequences," 1966.

[18] C. Bleakley and R. Scaife, "New formulas for predicting the accuracy of acoustical measurements made in noisy environments using the averaged m-sequence correlation technique," The Journal of the Acoustical Society of America, vol. 97, no. 2, pp. 1329-1332, 1995.

[19] T. Schneider and D. G. Jamieson, "A dual-channel MLS-based test system for hearing-aid characterization," Journal of the Audio Engineering Society, vol. 41, no. 7/8, pp. 583-594, 1993.

[20] P. Kovitz, "Two Maximal Length Sequence Devices for Measuring Room Acoustics Parameters," in Audio Engineering Society Conference: 11th International Conference: Test Eamp; Measurement, Audio Engineering Society, 1992.

[21] W. Ahnert, S. Feistel, and W. Richert, "Merging Room-acoustic and Electro-acoustic Measurement Methods," in Audio Engineering Society Convention 116, Audio Engineering Society, 2004.

[22] D. Griesinger, "Beyond MLS-Occupied hall measurement with FFT techniques," in Audio Engineering Society Convention 101, Audio Engineering Society, 1996.

[23] M. R. Bai and J. Liao, "Acoustic analysis and design of miniature loudspeakers for mobile phones," Journal of the Audio Engineering Society, vol. 53, no. 11, pp. 1061-1076, 2005. 


\section{APPENDIX}

The Discrete Fourier Transform (DFT) of a discrete periodic MLS $x[n]=x[n+L]$ with the period $L=2^{N}-1$ is given by

$$
X[k]=\left\{\begin{array}{ll}
\sqrt{\frac{1}{L}} e^{j \Phi_{k}}, & k=0 \\
\sqrt{\frac{L+1}{L}} e^{j \Phi_{k}}, & 1 \leq k \leq(L-1),
\end{array},\right.
$$

where $\Phi_{k}$ is a pseudo-random variable with uniform distribution on the range $[0,2 \pi]$. Without loss of generality, we shift the MLS $x[n]$ in time by $-(L-1) / 2$ samples to center it around zero, modify the DC component of the MLS $x[n]$ and multiply it by a constant in such a way that its DFT becomes

$$
X[k]=e^{j \Theta_{k}}, \quad 0 \leq k \leq(L-1),
$$

where the pseudo-random variable $\Theta_{k}$ has the same statistic properties as $\Phi_{k}$.

Suppose, that the MLS $x[n]$ (Fig. 4a) is converted to an analog signal using a perfect DAC working at sampling frequency $f_{s 1}$ (Fig. $4 \mathrm{~b}$ ) and then converted back to the digital sequence $y[n]$ using a perfect ADC working at sampling frequency $f_{s 2}>f_{s 1}$ (Fig. 4c) given by (Eq. 7), then the sequence $y[n]=y[n+L+\Delta L]$ is periodic with the period $L+\Delta L$, where $\Delta L$ is given in Eq. (6), and the DFT of the sequence $y[n]$ is

$$
Y[k]= \begin{cases}X[k], & 0 \leq k \leq \frac{L-1}{2} \\ 0, & \frac{L+1}{2} \leq k \leq\left(\frac{L-1}{2}+\Delta L\right) \\ X^{*}[k], & \left(\frac{L+1}{2}+\Delta L\right) \leq k \leq(L+\Delta L-1) .\end{cases}
$$

The sequence $y[n]$, being periodic with $L+\Delta L$ samples, is next truncated to $L$ samples and the $L$-point DFT $Y_{w}[k]$ is calculated (Fig. $4 \mathrm{~d}$ ). This can be mathematically described by two steps: multiplication by a finite length rectangular window and periodization of the truncated sequence.

The rectangular window of length $L$ and centered around zero is defined as

$$
w[n]= \begin{cases}1, & -\frac{L-1}{2} \leq n \leq \frac{L-1}{2} \\ 0, & \text { elsewhere }\end{cases}
$$

Consequently, the Discrete-time Fourier Transform (DtFT) $Y_{w}(f)$ of the time-limited sequence $y_{w}[n]=y[n] w[n]$ is given by

$$
Y_{w}(f)=\sum_{k=0}^{L+\Delta L} Y[k] \frac{\sin [\pi L(f-k \Delta f)]}{\sin [\pi(f-k \Delta f)]} .
$$

where

$$
\Delta f=\frac{f_{s 1}}{L}=\frac{f_{s 2}}{L+\Delta L} .
$$

The periodization of the truncated sequence $y_{w}[n]$ in the time domain over the period $L / f_{s 2}$ is equivalent to the discretization in the frequency domain with the DFT sample spacing of $f_{s 2} / L \mathrm{~Hz}$, expressed as

$$
Y_{w}[k]=\sum_{l=0}^{L+\Delta L} Y[l] \frac{\sin \left[\pi f_{s 2}\left(k-l \frac{L}{L+\Delta L}\right)\right]}{\sin \left[\pi \frac{f_{s 2}}{L}\left(k-l \frac{L}{L+\Delta L}\right)\right]},
$$




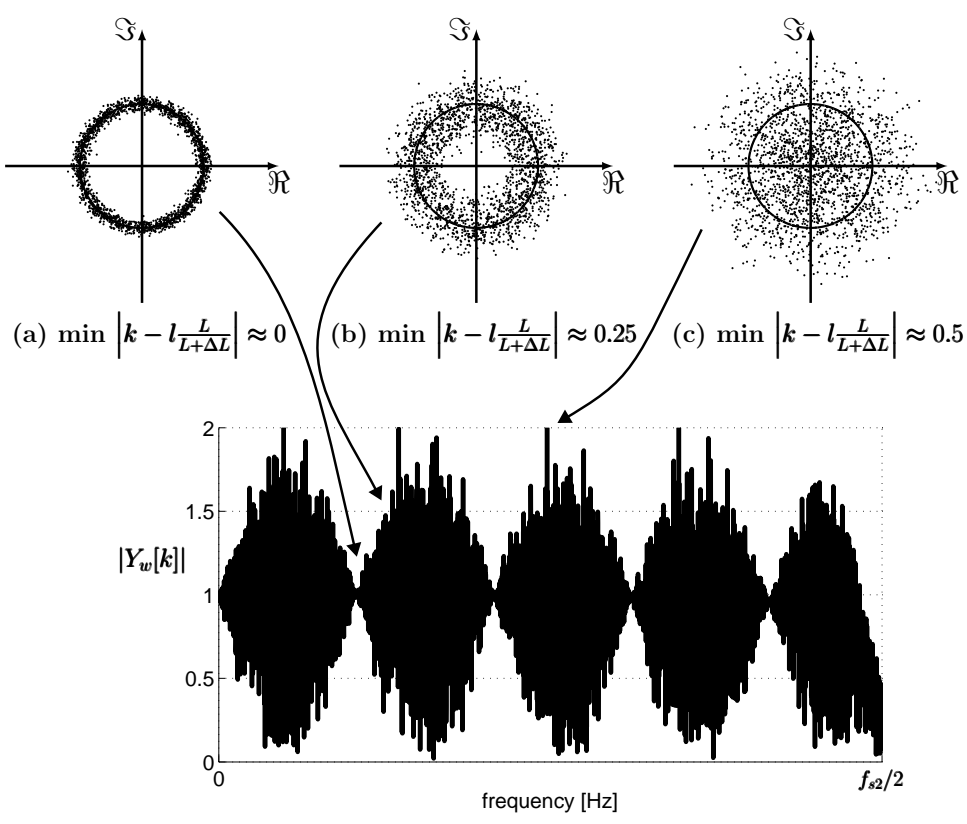

(d) DFT of $\mathrm{y}[\mathrm{n}]$ truncated to L samples

Figure 10. A graphical interpretation of pseudo-random distribution of $\left|Y_{w}(f)\right|$. The total sum over $k$ from

Eq. (A.5) has a distribution dependent on $l$ leading to a sine-like pattern in the spectra of the truncated sequence $y[n]$.

The statistic properties of $Y_{w}[k]$ are given by the sum of $Y[k]$ weighted by the sinc function of Eq. (A.7). The values of $Y[k]$ are distributed uniformly around a unit circle (Eqs. (A.2) and (A.3)). For some values of $k$, for which $\min _{l}|k-l L /(L+\Delta L)| \approx 0$, the sinc function approaches a delta function and the weighted sum is given mainly by one value of $Y[k]$ leading to a distribution of $Y_{w}[k]$ around the unit circle (Fig. 10a). On the other hand, for values of $k$ for which $\min _{l}|k-l L /(L+\Delta L)| \approx 0.5$, the weighting by the sinc function is spread over several values of $Y[k]$ leading to a distribution of $Y_{w}[k]$ around the unit circle with higher standard deviation (Fig. 10c). Consequently, there is a sine-like pattern in the spectra $Y_{w}[k]$ of the truncated sequence $y[n]$ characteristic for the asynchronous mode in MLS technique. Note that the spectra depicted in Fig. 3b has the same pattern as the one in Fig. 10d, except that one is plotted with a logarithmic axis and the other with a linear one. 